\title{
Hyperspectral Discrimination and Response Characteristics of Stressed Rice Leaves Caused by Rice Leaf Folder
}

\author{
Zhanyu Liu ${ }^{1,2, *}$, Jia-an Cheng ${ }^{2}$, Wenjiang Huang ${ }^{3}$, Cunjun $\mathrm{Li}^{3}$, Xingang $\mathrm{Xu}^{3}$, \\ Xiaodong Ding ${ }^{1}$, Jingjing Shi ${ }^{4}$, and Bin Zhou ${ }^{1}$ \\ ${ }^{1}$ Institute of Remote Sensing and Earth Sciences, Hangzhou Normal University, \\ Hangzhou 311121, China \\ ${ }^{2}$ Department of Plant Protection, Zhejiang University, Hangzhou 310029, China \\ ${ }^{3}$ Beijing Research Center for Information Technology in Agriculture, Beijing 100097, China \\ ${ }^{4}$ Institute of Agricultural Remote Sensing \& Information Technology, Zhejiang University, \\ Hangzhou 310029, China \\ liuzhanyudzju.edu.cn
}

\begin{abstract}
Detecting plant health condition plays an important role in controlling disease and insect pest stresses in agricultural crops. In this study, we applied support vector classification machine (SVC) and principal components analysis (PCA) techniques for discriminating and classifying the normal and stressed paddy rice (Oryza sativa L.) leaves caused by rice leaf folder (Cnaphalocrocis medinalis Guen). The hyperspectral reflectance of paddy rice leaves was measured through the full wavelength range from 350 to $2500 \mathrm{~nm}$ under the laboratory condition. The hyperspectral response characteristic analysis of rice leaves indicated that the stressed leaves presented a higher reflectance in the visible $(430 \sim 470 \mathrm{~nm}, 490 \sim 610 \mathrm{~nm}$ and $610 \sim 680 \mathrm{~nm}$ ) and one shortwave infrared $(2080 \sim 2350 \mathrm{~nm})$ region, and a lower reflectance in the near infrared $(780 \sim 890 \mathrm{~nm})$ and the other shortwave infrared (1580 1750 $\mathrm{nm}$ ) region than the normal leaves. PCA was performed to obtain the principal components (PCs) derived from the raw and first derivative reflectance (FDR) spectra. The nonlinear support vector classification machine (referred to as $C$ SVC) was employed to differentiate the normal and stressed leaves with the front several PCs as the independent variables of $C$-SVC model. Classification accuracy was evaluated using overall accuracy (OA) and Kappa coefficient. OA of $C$-SVC with PCA derived from both the raw and FDR spectra for the testing dataset were $100 \%$, and the corresponding Kappa coefficients were 1. Our results would suggest that it's capable of discriminating the stressed rice leaves from normal ones using hyperspectral remote sensing data under the laboratory condition.
\end{abstract}

Keywords: Hyperspectral remote sensing, Rice crop, Rice leaf folder, Principal components analysis (PCA), Support vector classification (SVC).

* Corresponding author.

D. Li and Y. Chen (Eds.): CCTA 2011, Part II, IFIP AICT 369, pp. 528-537, 2012.

(C) IFIP International Federation for Information Processing 2012 


\section{Introduction}

Detecting plant health condition plays an important role in controlling disease and insect pest stresses in agricultural crops, which always lead to yield loss and poor quality [1]. Although pest damage is predominantly concentrated in patches around original foci, the common protecting practice is still to spray agrochemicals indiscriminately over the entire fields [2]. To minimize economic loss and reduce ecological and environmental pressure due to the superfluous input of agrochemicals, it's necessary to accurately know the pest distribution locations and damage degrees caused by disease and insects [3] so that the most economical amount of agrochemicals can be applied to the infestation patches. It's a pity that the most common method of damage quantification evaluation is still visual survey [4]. However, this technique is subjective, laborintensive, and time-consuming, and it is impossible to accurately estimate the damaged areas and severity over a wide range [5]. Recently, remote sensing techniques have been proved to be effective to monitor insect infestation and disease epidemic in agricultural crops and other plants from ground to satellite platforms, especially in the site-specific crop management of precision agriculture [6].

The current studies have shown that plant spectral properties at visible and nearinfrared (NIR) regions vary with the development of specific signatures for a specific stress of a given plant species [2]. The reflectance or digital number of healthy plants is low in the visible region $(400 \sim 700 \mathrm{~nm})$ due to strong pigment absorption, is high in the NIR region (750 1300 nm) for cellular and subcellular structure, and is variable in the shortwave infrared (SWIR) region $(1300 \sim 2500 \mathrm{~nm}$ ) because of the presence of water vapor, proteins, and other biophysical and biochemical elements [2-3, 5]. In addition to raw reflectance, derivative spectral reflectance and various vegetation indices can also be effective in detecting plant stresses [6-7].

Furthermore, various statistical methods, including cluster analysis [8], decision tree [9], partial-least square regression [10], and principal component analysis (PCA) [2], have been used with various remote sensing data for detecting plant stresses. Among them, PCA is the most widespread method for hyperspectral remote sensing data because it can solve the high dependence and autocorrelation in adjacent wavebands [11]. Moreover, the application of artificial intelligence methods including artificial neural networks (ANN) and support vector machines (SVM) in plant stress detection has also been reported recently [2,12]. The nonlinear support vector classification machine (viz. $C$-SVC) had been used to detect the rice health condition at the leaf and canopy levels, which obtained above $85 \%$ of the classification accuracy [12].

Rice (Oryza sativa L.) is the most important staple food in Asia area and there have been a lot of remote sensing-based researches in rice disease and insect pest damage which harm the leaf lamina, leaf sheath, stem, panicles and other organs [2,12]. However, little study was found to discriminate the normal and stressed rice leaves caused by rice leaf folder [13-14]. Rice leaf folder (Cnaphalocrocis medinalis Guen) is widely distributed between $48^{\circ} \mathrm{N}$ and $24^{\circ} \mathrm{S}$ latitude and $0^{\circ} \mathrm{E}$ to $172^{\circ} \mathrm{W}$ longitude in the rice growing areas of Asia, Oceania, Australia and Africa [15]. The feeding damage caused by rice leaf folder shows up characteristically in pale stripe in the leaf where the chlorophyll-bearing tissues have been eaten away, and only epidermis and cuticle remains. All feeding damage is strictly linear, i.e., occurs in the longitudinal axis of the leaf between the veins. 
The purposes of the present study are (1) to analyze the hyperspectral response characteristics of raw and first derivative reflectance (FDR) of normal and stressed paddy rice leaves caused by rice leaf folder, (2) to identify the principal components (PCs) derived from both the raw and FDR spectra, and (3) to examine the capability of SVM in combination with PCA for discriminating normal and stressed rice leaves.

\section{Methodology}

\subsection{Study Sites}

The samples of normal and stressed rice leaves caused by rice leaf folder (Cnaphalocrocis medinalis Guen) were collected in three paddy rice fields at the active tillering stage. One field was located near Yundong village $\left(30^{\circ} 12^{\prime} \mathrm{N}\right.$, $\left.120^{\circ} 28^{\prime} \mathrm{E}\right)$, and the second in Zhangpanqiao Village $\left(30^{\circ} 06^{\prime} \mathrm{N}, 120^{\circ} 15^{\prime} \mathrm{E}\right)$, Xiaoshan District, and the last in the Experiment Farmland of Zhejiang University $\left(30^{\circ} 14^{\prime} \mathrm{N}\right.$, $\left.120^{\circ} 10^{\prime} \mathrm{E}\right)$, China. The sampling date in the former two paddy fields were on July $27^{\text {th }}$, 2007 and the sampling number of normal and stressed rice leaves in all was as equal as 19. The sampling date in the latter and last paddy field was on August $14^{\text {th }}, 2007$ and the sampling number of normal and stressed rice leaves was as equal as 35 . The rice cultivar names in the three sites were unknown, but all of them belonged to hybrid and Japonica rice.

\subsection{Foliar Hyperspectral Reflectance Measurement}

The normal and stressed rice leaves caused by rice leaf folder (Cnaphalocrocis medinalis Guen), which were verified by plant protection expert, were cut from the rice individuals in the paddy fields. The samples were put into an incubator $\left(25^{\circ} \mathrm{C}\right)$ and were transported to the laboratory for further measurements. The foliar hyperspectral reflectance of paddy rice was measured with a portable spectrophotometer (Analytical Spectral Devices Inc., Boulder, CO, USA) through the full wavelength range from $350 \mathrm{~nm}$ to $2500 \mathrm{~nm}$. The spectral resolution of the instrument varied from $3 \mathrm{~nm}$ less than $1000 \mathrm{~nm}$ to $10 \mathrm{~nm}$ more than $1000 \mathrm{~nm}$, while the spectra were interpolated by the spectrometer software in $1 \mathrm{~nm}$ intervals.

Each leaf was positioned on a dark background. The fiber optic sensor with $8^{\circ}$ instantaneous field of view (IFOV) vertically pointed to the upper leaf surface about 3 $\mathrm{cm}$ height. An incidence angle of $45^{\circ}$ was maintained at a standard distance $(50 \mathrm{~cm})$. The light source used was one 50 watt halogen lamp. For each paddy rice leaf, a sampling spectrum was made consisting of five readings and four sampling spectra were measured. The average hyperspectra derived from four sampling spectra represented each leaf using ViewSpec Pro (version 5.6.10). All spectral measurements were made relative to a $\mathrm{BaSO} 4$ standard reference-panel, which was observed immediately before and after the spectral measurements of rice leaves.

\subsection{Foliar Pigment Concentration Measurement}

Immediately after foliar hyperspectral reflectance measurements have been taken, the leaves were chipped, weighted and then dipped in 20 milliliter solution (acetone: 
ethanol: distilled water $=4.5: 4.5: 1)$ for 24 hours to extract pigment. The optical density of the extraction solution was measured at $440 \mathrm{~nm}, 645 \mathrm{~nm}$, and $663 \mathrm{~nm}$ by spectrophotometer (Shimadzu UV 2550, Tokyo, Japan). Pigment consists of chlorophyll a (ab. Chl a), Chlorophyll b (ab. Chl b), total chlorophyll (ab. ChlT, which is equal to $\mathrm{Chl}(\mathrm{a}+\mathrm{b})$ ) and Carotenoid (ab. Car) in the study. The formula of pigment concentration $(\mathrm{mg} / \mathrm{g})$ was referenced the literature of Tang et al. [16]. The statistical information of pigment on rice leaves was shown in Table 1.

\subsection{Data Preprocessing}

Firstly, the hyperspectral reflectance was smoothed with a seven step moving average to suppress the instrumental and environmental noise before these data were further analyzed [5]. Next, the raw hyperspectral reflectance less than $400 \mathrm{~nm}$ and more than $2400 \mathrm{~nm}$ were deleted due to severe instrument and system noise. The effective spectral region of FDR ranged from $450 \mathrm{~nm}$ to $850 \mathrm{~nm}$. Furthermore, the total dataset was consciously divided into two parts, namely training and testing datasets. The training dataset included the normal and stressed rice leaves caused by rice leaf folder, viz. those leaves which were collected on August $14^{\text {th }}, 2007$. The testing dataset was consisted of those leaves which were collected on July $27^{\text {th }}, 2007$.

Derivative spectroscopy is proposed to tackle analogous problems such as background signals and to solve overlapping problems in spectral features. In practice, the simplest method of finding derivatives is by dividing the difference between successive spectral reflectance by the wavelength interval separating them [7]. The FDR spectra can provide the slope information on the rate of change reflectance with respect to wavelength [17]. FDR were calculated to analyze the hyperspectral response characteristics of stressed rice leaves caused by rice leaf folder in this study.

\subsection{Analytical Techniques}

Principal component analysis (PCA) is capable of preserving the total variance and minimizing the mean square approximate errors, and it is also used to identify the dominant modes of the original dataset such that the first principal component $\left(\mathrm{PC}_{1}\right)$ accounts for the maximum possible proportion of the variance information of the original dataset, and subsequent principal components $\left(\mathrm{PC}_{2}, \mathrm{PC}_{3} \ldots\right)$ account for the maximum proportion of the unexplained residual variance, and so forth [17]. The application of PCA has been reported in many studies as an effective data compression technique especially for hyperspectral remote sensing data because it can solve the high dependence and autocorrelation in adjacent wavebands [11]. PCs were calculated to represent the hyperspectral features of rice leaves in this study.

Support vector classification (SVC), which is a popular technique for data classification, is one of the most important parts of support vector machine (SVM). The aim of SVC is to devise a computationally efficient way of learning 'good' separating hyperplanes in a high dimensional feature space based on the generalization and optimization theory [18]. SVC was conducted in LIBSVM (Library for Support Vector Machines) developed by Chang and Lin [19] in our study. The goal of LIBSVM is to help users to easily use SVM as an attached library 
for SVM. LIBSVM is consisted of $C$-SVC, $v$-SVC, distribution estimation (one-class $\mathrm{SVM}$ ), and other models. Thereinto $C$-SVC was adopted in this study. The key parameters of LIBSVM such as kernel function $\gamma$ and penalty parameter $C$ and other parameters are provided with default selections available.

There were two assessment parameters to evaluate the accuracy of C-SVC combing with PCA in this study. One is Kappa coefficient which is one measure of the level of agreement. A Kappa coefficient of 1 denotes a full agreement and 0 denotes full disagreement between the actual and predictive classification [20]. The other is overall accuracy (OA) which denotes the agreement probability between the predictive and actual classification for each random sample.

\section{$3 \quad$ Results}

\subsection{Hyperspectral Response Characteristics of Paddy Rice Leaves}

Applying hyperspectral remote sensing to precise crop integrated pest management (IPM) is on the basis of spectral response characteristics of paddy rice leaves to crop insect stress in Fig.1. Fig.1 showed the hyperspectral difference was obvious between normal and stressed paddy rice leaves caused by rice leaf folder at the nanometer level because there were evident changes in pigment concentration (Table 1), cell and texture arrangement structure and foliar water content due to the feeding damage caused by rice leaf folder [15]. Furthermore, six wavelength intervals (Table 2) had been calculated to simulate the high resolution geometric (HRG) and vegetation (VGT) sensors of Systeme Probatoire d'Observation dela Tarre (SPOT) satellite based on arithmetic mean of hyperspectral reflectance for universal application of SPOT's image in vegetation monitoring all over the world. The simulated six wavelength intervals clearly indicated the hyperspectral difference between normal and stressed paddy rice leaves from visible spectrum region (PA: 460 690 nm, B0: 430 470 nm, B1: 490 610 $\mathrm{nm}$ and B2: 610 680 nm) to NIR (B3: 780 890 nm) and SWIR (1580 1750 nm).

As shown in Fig.1 (a), the raw hyperspectral feature of the normal paddy rice leaves was different from the stressed leaves through the full spectra range (400 2400 $\mathrm{nm}$ ). Because paddy rice foliar pigment concentration decreased obviously (Table 1), hyperspectral reflectance of stressed leaves increased 2.9, 1.9 and 3.3 times than the normal ones in the visible blue (B0), green and near red (B1) and red (B2) light region (Table 2), respectively. However, the hyperspectral reflectance of stressed leaves decreased $20.7 \%$ and $9.7 \%$ than the normal ones in NIR (B3) and one SWIR $(2.08 \sim 2.35 \mu \mathrm{m})$ region, respectively. But in the other SWIR region, the hyperspectral reflectance increased $28.9 \%$ in comparison with the normal ones.

The amplitude of FDR for stressed paddy rice leaves was smaller than that of normal ones as shown in Fig.1 (b). Maybe it's because the stressed paddy rice leaves looked like 'white' color after rice leaf folder fed; the hyperspectral reflectance of the stressed paddy rice leaves had lower change rage with respect to wavelengths. Both of the peak wavebands of normal and stressed leaves in the blue light region were located at $521 \mathrm{~nm}$. Same phenomenon occurred in the green light region, the peak waveband $(569 \mathrm{~nm})$ of stressed leaves was equal to normal leaves. The red edge position of normal leaves was at $702 \mathrm{~nm}$, but the stressed leaves shifted toward short wavebands about $5 \mathrm{~nm}$. 


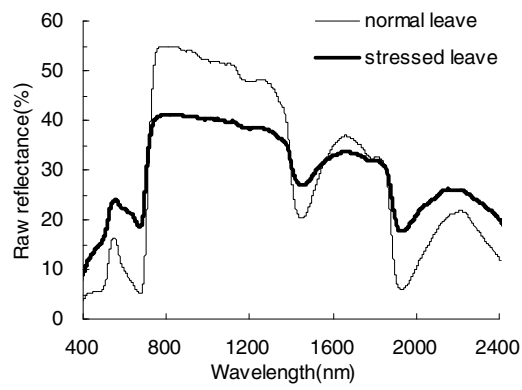

(a)

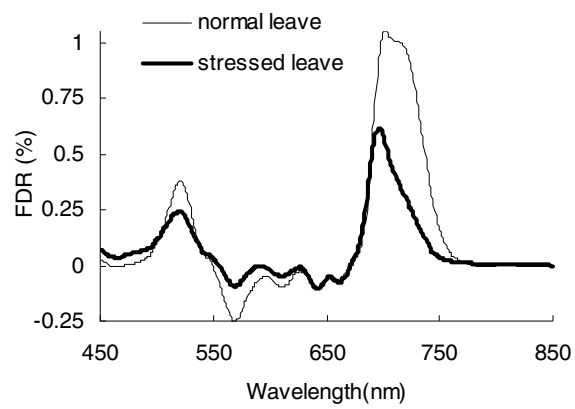

(b)

Fig. 1. Average hyperspectral reflectance of rice leaves collected on August $14^{\text {th }}, 2007$

Table 1. Statistical description of paddy rice foliar pigment concentration (Unit: $\mathrm{mg} / \mathrm{g}$ )*

\begin{tabular}{lcccccc}
\hline Statistical parameters & \multicolumn{3}{c}{ Normal rice leaves, N=35 } & \multicolumn{3}{c}{ Stressed rice leaves, N=35 } \\
\cline { 2 - 7 } & Chl a & Chl b & Car & Chl a & Chl b & Car \\
\hline Min. & 1.63 & 0.57 & 0.60 & 0.88 & 0.36 & 0.35 \\
Mean & 2.06 & 0.71 & 0.76 & 1.35 & 0.52 & 0.54 \\
Max. & 2.53 & 0.83 & 0.96 & 1.90 & 0.73 & 0.79 \\
Std. dev & 0.19 & 0.06 & 0.08 & 0.25 & 0.10 & 0.10 \\
VC & $9.2 \%$ & $8.0 \%$ & $10.1 \%$ & $18.7 \%$ & $18.7 \%$ & $19.4 \%$ \\
\hline
\end{tabular}

* The paddy rice leaves were collected on August $14^{\text {th }}, 2007$.

Table 2. Average canopy hyperspectral reflectance of rice crops at six wavelength intervals representing the High Resolution Geometric (HRG) and Vegetation (VGT) of SPOT (Unit: \%)*

\begin{tabular}{ccccc}
\hline \multirow{2}{*}{ Spectra range } & \multicolumn{2}{c}{ July $27^{\text {th }}, 2007$} & \multicolumn{2}{c}{ August $14^{\text {th }}, 2007$} \\
\cline { 2 - 5 } & Normal(n=19) & Stressed(n=19) & Normal(N=35) & Stressed(n=35) \\
\hline Pan: 490 690 nm & $10.1 \mathrm{c}$ & $22.6 \mathrm{a}$ & $9.6 \mathrm{c}$ & $20.9 \mathrm{~b}$ \\
B0: 430 470 nm & $5.2 \mathrm{c}$ & $15.0 \mathrm{a}$ & $5.5 \mathrm{c}$ & $13.4 \mathrm{~b}$ \\
B1: 490 610 nm & $12.3 \mathrm{c}$ & $22.8 \mathrm{a}$ & $11.6 \mathrm{c}$ & $21.4 \mathrm{~b}$ \\
B2: $610 \sim 680 \mathrm{~nm}$ & $6.8 \mathrm{c}$ & $22.3 \mathrm{a}$ & $6.7 \mathrm{c}$ & $20.1 \mathrm{~b}$ \\
B3: 780 890 nm & $57.9 \mathrm{a}$ & $45.9 \mathrm{c}$ & $54.7 \mathrm{~b}$ & $41.1 \mathrm{~d}$ \\
SWIR: $1580 \sim 1750 \mathrm{~nm}$ & $39.2 \mathrm{a}$ & $35.4 \mathrm{~b}$ & $35.7 \mathrm{~b}$ & $33.2 \mathrm{c}$ \\
\hline
\end{tabular}

* Reflectance values within a row followed by the same letter are not significantly different from each other by Duncan test at $\alpha=0.05$.

\subsection{Principal Component Analysis}

The first principal component (PC1) explained $72.1 \%$ of the variance of the raw hyperspectral reflectance in rice leaves for the training dataset. Fig.2 showed both eigenvector of PC1 and the spectral reflectance difference between the stressed and normal leaves. PC1 had a loading of 0.958 to the reflectance difference of raw spectra between the stressed and normal rice leaves. 


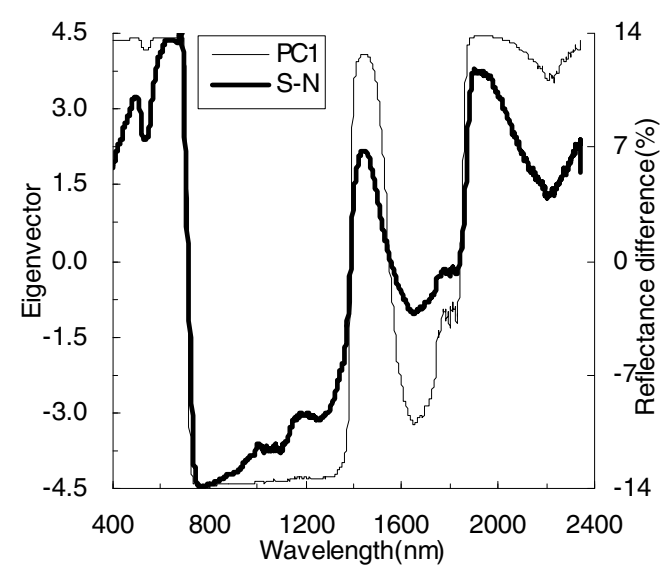

Fig. 2. Loading plot of the first principal component (PC1) derived from the raw hyperspectral reflectance. S-N meant the hyperspectral reflectance difference between the stressed and normal paddy rice leaves.

The results of the principal component analysis (PCA) revealed that the cumulative contribution of the front three components of the raw hyperspectral reflectance and FDR for the training dataset were $98.1 \%$ and $66.2 \%$, respectively (Table 3). Generally speaking, when the cumulative contribution of the front several components is above $80 \sim 85 \%$, the remaining components always are omitted in further analysis ${ }^{[17]}$. So the front eighteen components could explain $85.6 \%$ of the variance of FDR for training dataset, which were regarded as the principal component spectra (PCs) in this study.

Table 3. Percentage of explained variance for the front three PCs (Unit: \%)*

\begin{tabular}{ccccccccc}
\hline \multirow{2}{*}{$\begin{array}{c}\text { Spectral } \\
\text { types }\end{array}$} & \multicolumn{3}{c}{ Training dataset (August $\left.14^{\text {th }}, 2007\right)$} & \multicolumn{4}{c}{ Testing dataset (July 27 $\left.{ }^{\text {th }}, 2007\right)$} \\
\cline { 2 - 9 } & PC1 & PC2 & PC3 & CC & PC1 & PC2 & PC3 & C \\
\hline Raw & 72.1 & 24.6 & 1.4 & 98.1 & 70.4 & 26.8 & 1.6 & 98.8 \\
FDR & 47.9 & 14.1 & 4.2 & 66.2 & 68.4 & 11.1 & 7.6 & 87.2 \\
\hline
\end{tabular}

* CC denotes the cumulative contribution.

\subsection{Results of Support Vector Classification Machine}

The nonlinear support vector classification machine (referred to as $C$-SVC) was used to automatically detect the health condition of paddy rice leaves. The $C$-SVC model was built to differentiate the stressed rice leaves from normal ones by using the training dataset consisting of dependent (i.e. normal and stressed rice leaf categories) and independent (i.e. PCs) variables for calibration purpose. The $C$-SVC model was run repeatedly with multifarious sets of values of $\gamma$ (kernel function) and $C$ (penalty parameter) at least three times for cross-validation in order that the optimum classification result could be obtained. When $\gamma$ was RBF (radial basis function) and $C$ 
was 1 , the $C$-SVC model was able to discriminate paddy rice leaves categories without any error. Then the $C$-SVC model was validated by the testing dataset and the classification results were given in Table 4. Not only for the raw hyperspectral reflectance, but also FDR spectra, none of normal or stressed paddy rice leaf was classified mistakenly. The stressed rice leaves had obviously spectral difference from normal ones after the rice leaf folder fed (Fig. 1 and Table 2), which resulted in the perfect classification result by $C$-SVC model integrated with PCA.

Table 4. Error matrix for C-SVC from the testing dataset with PCs derived from raw and FDR spectra

\begin{tabular}{ccccccc}
\hline & \multicolumn{5}{c}{ Predictive classification } \\
\cline { 2 - 7 } $\begin{array}{c}\text { Actual } \\
\text { classification }\end{array}$ & \multicolumn{3}{c}{ Raw spectra } & \multicolumn{3}{c}{ FDR spectra } \\
& Normal & Stressed & Total & Normal & Stressed & Total \\
\cline { 2 - 7 } & 19 & 0 & 19 & 19 & 0 & 19 \\
\hline Normal & 0 & 19 & 19 & 0 & 19 & 19 \\
Stressed & 19 & 19 & 38 & 19 & 19 & 38 \\
Total & & & & & & \\
\hline
\end{tabular}

\section{Discussions}

In this study, $C$-SVC was employed to detect the health condition of paddy rice leaves by integrating it with PCA using raw hyperspectral reflectance and FDR. The results indicated that the stressed rice leaves caused by rice leaf folder presented a higher reflectance in the visible $(430 \sim 470 \mathrm{~nm}, 490 \sim 610 \mathrm{~nm}, 610 \sim 680 \mathrm{~nm})$ and one shortwave infrared $(2080 \sim 2350 \mathrm{~nm})$ region, and a lower reflectance in the NIR $(780 \sim 890 \mathrm{~nm})$ and the other SWIR $(1580 \sim 1750 \mathrm{~nm})$ region than the normal leaves (Fig.1a and Table 2). These results were consistent with our previous research [13], and were similar to other's results for geenbug-damaged wheat at the leaf or canopy level [6]. However, our results were not in agreement with the report from Huang et al. [14] who concluded that the spectral reflectance of rice leaf decreased in the green $(530 \sim 570 \mathrm{~nm})$ region with the increase of the damage degrees. Perhaps because in our study the rice leaf folder almost fed on the whole upper surface leaf, there were little pigment concentration in the remaining downer surface leaf than the stressed leaves caused by greenbug [6] or rice leaf folder [14] in others reports.

FDR spectra were also analyzed from a different view. Perhaps because the stressed paddy rice leaves looked like 'white' color after rice leaf folder fed, the amplitude of FDR for stressed leaves was smaller than that of normal ones (Fig.1 b). Not only the stressed paddy rice leaves, but also the normal ones, the peak wavebands of blue and green spectrum region were located at $521 \mathrm{~nm}$ and $569 \mathrm{~nm}$, respectively. But the stressed leaves shifted toward short wavebands about $5 \mathrm{~nm}$, which suggested that the red light spectrum region was more sensitive to plant pest damage than the blue and green regions. Similar results occurred in other results [6].

The front several PCs were found to be sensitive to the reflectance and digital number of healthy and nonhealthy plants as well as their linear combinations according to current research [2]. Same results were also found in this study. PC1 
could explain $95.8 \%$ of variance in the raw reflectance difference between the stressed and normal rice leaves (Fig.2). The front three PCs would reveal above $85 \%$ of cumulative contribution except the FDR spectra of training dataset (Table 3). Subsequently, the classification was almost perfect with the combination of $C$-SVC classifier and PCA for discriminating the stressed rice leaves from normal ones with the obtaining PCs (Table 4). Maybe it's because the stressed rice leaves had obviously spectral difference from normal ones after the rice leaf folder fed (Fig. 1 and Table 2).

\section{Conclusions}

Even though the present study would suggest that it's capable of discriminating the stressed rice leaves from normal ones using hyperspectral remote sensing data under the laboratory condition, this technique is probably still not very practical for detecting the health condition of agricultural crops under the field conditions [6]. More external factors, including atmospheric (e.g., illumination, cloudy shadow), edaphic (e.g., soil type, soil moisture), and biotic (e.g., crop variety, leaf area index) conditions as well as field management strategies, coexist at the canopy level [2], which consequentially bring about too much noise in hyperspectral reflectance data and lower classification accuracy for stressed crops and their organs. Therefore, more studies are needed to explore the spectral response characteristics of rice crops under fungi and insect stresses of different levels in outdoor conditions.

The samples of rice leaves only had two levels, namely, normal and stressed rice leaves caused y rice leaf folder. However, the rate of rolling leaf is employed to determine the damage severity caused by rice leaf folder in practice. Future study should include more stress levels in order to discriminate not only the normal rice leaves from the stressed ones but different stress status.

Acknowledgements. This research was supported by the China Postdoctoral Science Special Foundation Project (201003712), the Start Research and Fund Profect of Hangzhou Normal University (2011QDL23), the National Basic Research Program (973) of China (2010CB126200), the Agro-Industry R\&D Special Fund of China (200903051), and the National Hi-Tech Research and Development Program (863) of China (2007AA10Z205).

\section{References}

1. Everitt, J.H., Escobar, D.E., Summary, K.R.: Using airborne video, global positioning system, and geographical information system technologies for detecting and mapping citrus blackfly infestations. Southwest Entomol. 19(2), 129-138 (1994)

2. Liu, Z.Y., Wu, H.F., Huang, W.J.: Application of neural networks to discriminate fungal infection levels in rice panicles using hyperspectral reflectance and principal components analysis. Comput. Electron. Agr. 72(2), 99-106 (2010)

3. Pedigo, L.P.: Closing the gap between IPM theory and practice. J. Agr. Entomol. 12(4), 171-181 (1995) 
4. Moshou, D., Bravo, C., West, J., Wahlen, S.: Automatic detection of 'yellow rust' in wheat using reflectance measurements and neural networks. Comput. Electron. Agr. 44(3), 173-188 (2004)

5. Kobayashi, T., Kanda, E., Kitada, K.: Detection of rice panicle blast with multispectral radiometer and the potential of using airborne multispectral scanners. Phytopathol. 91(3), 316-323 (2001)

6. Yang, Z., Rao, M.N., Ellitott, N.C., Kindler, S.D.: Differentiating stress induced by greenbug and Russian wheat aphids in wheat using remote sensing. Comput. Electron. Agr. 67(1-2), 64-70 (2009)

7. Demetriades-Shah, T., Steven, M., Clark, J.: High- resolution derivative spectra in remote sensing. Remot. Sens. Environ. 33(1), 55-64 (1990)

8. Li, B., Liu, Z.Y., Huang, J.F.: Hyperspectral identification of rice diseases and pests based on PCA and PNN. Trans. CSAE 25(9), 143-148 (2009)

9. Goel, P.K., Prasher, S.O., Patel, R.M.: Classification of hyperspectral data by decision trees and artificial neural networks to identify weed stress and nitrogen status of corn. Comput. Electron. Agr. 39, 67-93 (2003)

10. Huang, J.F., Apan, A.: Detection of Sclerotinia rots disease on celery using hyperspectral data and partial least squares regression. J. Spat. Sci. 52(1), 131-144 (2006)

11. Panda, S.S., Hoogenboom, G., Paz, J.: Distinguishing blueberry bushes from mixed vegetation land use using high resolution satellite imagery and geospatial techniques. Comput. Electron. Agr. 67(1-2), 51-58 (2009)

12. Liu, Z.Y.: Monitoring the rice disease and insect stress with remote sensing. Doctor Thesis, pp. 79-87. Zhejiang University, Hangzhou, China (2008)

13. Shi, J.J., Liu, Z.Y., Zhang, L.L.: Hyperspectral recognition of rice damaged by rice leaf roller based on support vector machine. Rice Sci. 23(3), 331-334 (2009)

14. Huang, J.R., Sun, Q.H., Liu, X.D.: Spectral characteristics of rice leaves damaged by rice leaf roller. Sci. Agri. Sin. 43(13), 2679-2687 (2010)

15. Khan, Z.R., Barrion, A.T., Litsinger, J.A.: A bibliography of rice leaf-folders (Lepidoptera: Pyralidae). Insect Sci. Appl. 9(2), 129-174 (1988)

16. Tang, Y.L., Wang, R.C., Huang, J.F.: Relations between red edge characteristics and agronomic parameters of crops. Pedo. 14(4), 467-474 (2004)

17. Holden, H., LeDrew, E.: Spectral discrimination of healthy and non-healthy corals based on cluster analysis, principal component analysis, and derivative spectroscopy. Remot. Sens. Environ. 65(2), 217-224 (1998)

18. Cristianini, N., Shawe-Taylor, J.: An Introduction to Support Vector Machines and Other Kernel-Based Learning Methods, pp. 93-112. Cambridge University Press, London (2000)

19. Chang, C.C., Lin, C.J.: LIBSVM: a library for support vector machines. (2001) Software available at, http://www. csie.ntu.edu.tw/ cjlin/libsvm

20. Congalton, R.G.: A review of assessing the accuracy of classification of remotely sensed data. Remot. Sens. Environ. 37(1), 35-46 (1991) 